\title{
Frameworks for commercial success
}

\author{
Taking chemical technology from the bench to the consumer is a formidable challenge, but it is how \\ research can ultimately benefit wider society. Companies are now beginning to incorporate metal- \\ organic frameworks into commercial products, heralding a new era for the field.
}

Metal-organic frameworks (MOFs) have risen to prominence in the chemical literature over the past 20 years, to the extent that they now barely need an introduction. This family of materials which can be most simply described as infinite networks of metal ions or clusters held together by organic linkers - first garnered attention on account of the ultrahigh porosity and surface area that some of them exhibit. Together with great scope for chemical functionalization, such properties immediately suggested possible future applications in gas storage, separation and catalysis. Indeed, research has since developed to the point where there are now a broad array of exciting candidates for implementation in real-world systems.

The potential capabilities of these materials are frequently cited in grant applications and scientific papers. Indeed, there are credible claims that MOF-enabled technologies can resolve some of the grand challenges of our age - particularly those associated with issues concerning energy and the environment. These include carbon capture as a means to curb climate change, low-pressure gas storage for portable fuels (especially relevant for a feasible hydrogen economy) and catalytic break down of toxic species to name a few. And yet there is a growing contingent of naysayers who suggest that MOFs have promised much and delivered little, and furthermore that the barriers to the realization of genuine MOF-based solutions to these problems are insurmountable.

Contrary to this perception, some companies have already demonstrated strong proof-ofconcept studies and, more recently, have even released products to the market. The chemical company BASF took an interest in MOFs following high-profile publications in Science ${ }^{1}$ and Nature ${ }^{2}$ in 1999; they soon struck up a collaboration with Omar Yaghi, currently at the University of California, Berkeley. As a result of this partnership, BASF developed a MOF-based natural gas storage system which, since 2013, they have been testing in a fleet of demonstration vehicles ${ }^{3}$. Although economic pressures may not currently favour methane fuels, indications are that this technology is getting very close to the market ${ }^{4}$.

In July of this year, Decco announced $^{5}$ the registration of TruPick - a post-harvest freshness management tool for fruit and vegetables with the US Environmental Protection Agency. Details of the product were revealed ${ }^{6}$ in September at the 5th International Conference on Metal-Organic Frameworks \& Open Framework Compounds (MOF2016) in California. The product, enabled by their partner MOF Technologies - a spin-out company from Queens University Belfast — uses a MOF adsorbent for the storage and release of 1-methylcyclopropene (1-MCP), which is a competitive inhibitor for the ethylene receptor found on the surface of some fruit. In large storage facilities, ethylene is released by ripening fruit, which in turn accelerates the ripening process in the surrounding fruit and can cause significant problems with premature aging. The strong binding of 1-MCP can, however, significantly prolong the time for which fruit can be safely stored by up to nine months. TruPick is already being used in the USA and our American readers could be eating September's harvest of apples in March next year; registration in other countries is expected soon, with Turkey's having already been received. MOF Technologies are now in the process of scaling up their solvent-free mechanochemical production capabilities to $100 \mathrm{~kg}$ per week across a range of MOFs.

Rather than act as a MOF provider, NuMat Technologies - a company spun out of Northwestern University - took a different approach and chose to deliver a complete MOF-integrated product ready for the market. Also at MOF2016, NuMat announced the launch of a system for the storage of hazardous gases that are typically used in the electronics industry. During the conference, ION-X cylinders were already on their way to customers. We interview Omar Farha and Ben Hernandez from NuMat on page 990 of this issue, where you can read more about how the company came about, and what strategies they needed to implement to get to this point.

With at least two companies now taking products to market, it seems that a critical developmental stage has been reached in the maturity of MOF research. There remain many hurdles before some of the most globally significant goals can be reached, but these early footsteps by MOF pioneers into the brutally competitive arena of technology commercialization will surely pave the way for others to do the same. Moreover, by following through on promises to make MOF-enabled products that can deliver tangible societal benefits - as well as the sustainability that comes with commercial revenue - the example set by the scientists and engineers behind these companies should encourage funding agencies and private investors to continue to support research in this area.

References

1. Chui, S. S.-Y., Lo, S. M.-F., Charmant, J. P. H., Orpen, A. G. \& Williams, I. D. Science 283, 1148-1150 (1999).

2. Li, H., Eddaoudi, M., O'Keeffe, M. \& Yaghi, O. M. Nature 402, 276-279 (1999).

3. Chem. Soc. Rev. 43, 6173-6174 (2014).

4. Peplow, M. Nature 520, 148-150 (2014).

5. Decco/ESSENTIV ${ }^{m}$ Announce US EPA Registration of TruPick for Postharvest Freshness Management (Decco, 2016); http://www.deccopostharvest.com/pdf/newsletter/12_07_2016/ EPA-registration-of-TruPick-12072016.pdf

6. MOF Technologies Announces World's First Commercial Application of Metal Organic Framework Technology by Decco Worldwide at MOF 2016 (MOF Technologies); http://www. moftechnologies.com/2016/09/13/mof-announces-worlds-firstcommercial-application-decco-worldwide-mof-2016/ 